\title{
A view of the implanted SiC damage by Rutherford backscattering spectroscopy, spectroscopic ellipsometry, and transmission electron microscopy
}

\author{
G. Battistig, ${ }^{\text {a) }}$ N. Q. Khánh, P. Petrik, T. Lohner, L. Dobos, and B. Pécz \\ Research Institute for Technical Physics and Materials Science, Konkoly Thege Miklós út 29-33, H-1121 \\ Budapest, Hungary \\ J. García López and Y. Morilla \\ Centro Nacional de Aceleradores, Avda. Thomas A. Edison s/n, E-41092 Sevilla, Spain
}

(Received 14 February 2006; accepted 8 August 2006; published online 3 November 2006)

\begin{abstract}
$4 H$-SiC single crystalline substrates were implanted at room temperature with $150 \mathrm{keV} \mathrm{Al}{ }^{+}$ions using fluences of $4 \times 10^{14}, 1 \times 10^{15}$, and $2 \times 10^{15} \mathrm{~cm}^{-2}$ with current density of $2.5 \mu \mathrm{A} \mathrm{cm}^{-2}$. The samples were subsequently annealed at $1100{ }^{\circ} \mathrm{C}$ in $\mathrm{N}_{2}$ for $1 \mathrm{~h}$ in order to analyze their structural recovery. The disorder induced in both sublattices by the $\mathrm{Al}^{+}$ions was studied by backscattering spectrometry in channeling geometry with a $3.5 \mathrm{MeV} \mathrm{He}^{2+}$ beam. The results were compared with the optical properties of the samples measured by spectroscopic ellipsometry. In a previous work, we concluded that during the postimplantation annealing of a highly damaged $\mathrm{SiC}$ crystalline material the short distance order can be recovered, while the long distance disorder remains. We also presented the possibility to have grains of different polytypes oriented faraway from the original direction. Now, this alternative is confirmed by the cross-sectional transmission and high resolution electron microscopy studies, carried out to obtain information about the crystal structure. (C) 2006 American Institute of Physics. [DOI: 10.1063/1.2360150]
\end{abstract}

\section{INTRODUCTION}

It is an accepted idea that the devices produced by doping $\mathrm{SiC}$, with different elements, have a great prospective for an extensive range of applications involving high temperature, high power, and high frequency. ${ }^{1}$ However, the local doping in $\mathrm{SiC}$ can only be performed by ion implantation due to the very low diffusivity of the dopants in this material even at high temperature. The disorder induced by the implantation process in this semiconductor depends not only on the energy and fluence of the implanted ions but also on the ion current during the implantation. ${ }^{2-4}$ The material must be annealed subsequently to recuperate its crystal lattice and to activate the dopants. Due to the importance for the future manufacture of this kind of devices, the lattice damage and its behavior under different annealing conditions has been extensively studied by several techniques, especially transmission electron microscopy (TEM) and Rutherford backscattering spectrometry in channeling geometry (RBS/C). Nondestructive optical techniques, such as spectroscopic ellipsometry (SE), can also give important information about this problem, since the ion implantation changes the refractive index of $\mathrm{SiC}^{3,5,6}$

This paper addresses a comparative study of the disorder in $4 H$-SiC after $\mathrm{Al}$ implantation at different fluences by RBS/C, SE, TEM, and high resolution electron microscopy (HREM). Moreover, the effect of thermal annealing in the recovery of defects and recrystallization of the samples is studied.

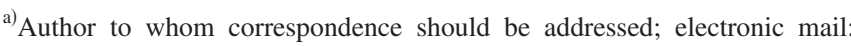
battisti@mfa.kfki.hu
}

\section{EXPERIMENT}

n-type single crystalline $\langle 0001\rangle 4 H$-SiC wafers (Cree Res., Inc.) were implanted at room temperature with $150 \mathrm{keV} \mathrm{Al}$ ions using fluences of $4 \times 10^{14}, 1 \times 10^{15}$, and 2 $\times 10^{15} \mathrm{~cm}^{-2}$ with current density of $2.5 \mu \mathrm{A} \mathrm{cm} \mathrm{c}^{-2}$. A postimplantation annealing was performed at $1100{ }^{\circ} \mathrm{C}$ in $\mathrm{N}_{2}$ ambient for $1 \mathrm{~h}$. The crystal quality of the samples was analyzed by RBS/C along the $\langle 0001\rangle$ axial channeling direction, SE, and TEM-HREM before and after the annealing.

The RBS/C measurements were performed employing a $3.5 \mathrm{MeV}{ }^{4} \mathrm{He}^{2+}$ beam, with the surface barrier detector placed at $165^{\circ}$ in Cornell geometry. To avoid the additional damage created by the analyzing beam itself ${ }^{7,8}$ a low current of $5 \mathrm{nA}$ was used during the experiments. Non-Rutherford backscattering spectrometry in channeling geometry (BS/C) using a $3.5 \mathrm{MeV}{ }^{4} \mathrm{He}^{2+}$ beam is an appropriate method to study simultaneously both $\mathrm{C}$ and $\mathrm{Si}$ sublattices, since an enhancement in the cross section for carbon about six times larger than the Rutherford one ${ }^{3,9}$ appears. The BS/C spectra were evaluated by means of the RBX simulation code. ${ }^{10}$

The SE experiments were carried out with a Sopra ES4G rotating polarizer spectroscopic ellipsometer at an angle of incidence of $75.1^{\circ}$, in the wavelength range of $280-840 \mathrm{~nm}$, with $10 \mathrm{~nm}$ steps. Details on the RBS/C and the SE measurements on these specimens are given in Refs. 3 and 5 .

Cross-sectional samples for TEM analysis have been prepared by cutting the samples and embedding the pieces face to face in a special Ti grid. The embedded samples were ground with diamond paste and subsequently thinned by ion milling at $10 \mathrm{keV}$ by $\mathrm{Ar}^{+}$ions. In order to minimize the ion beam damage the bombarding ion energy was decreased to 


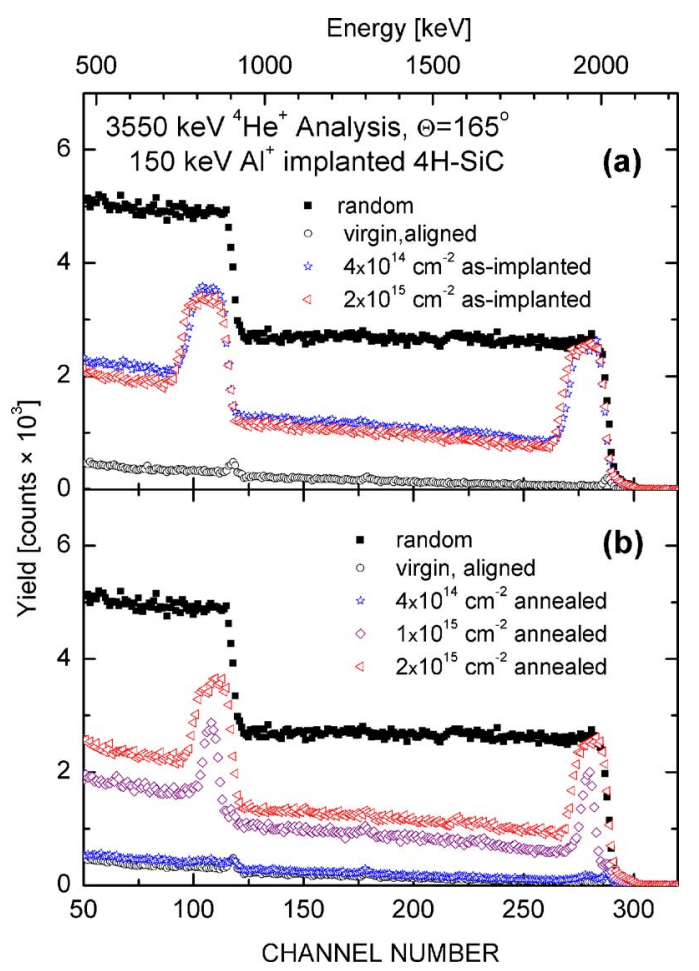

FIG. 1. (Color online) BS spectra taken on ion implanted $4 H$-SiC by $3550 \mathrm{keV} \mathrm{He}{ }^{+}$beam at channeling direction. Due to the elevated cross section of carbon at this energy, the disorder in the carbon sublattice is also well visible (from channels 90 to 140 approximately). The samples were implanted by $150 \mathrm{keV} \mathrm{Al}$ ions with fluences of $4 \times 10^{14}, 1 \times 10^{15}$, and 2 $\times 10^{15} \mathrm{~cm}^{-2}$ with ion current density of $2.5 \mu \mathrm{A} \mathrm{cm}^{-2}$. Spectra recorded on (a) as-implanted and (b) annealed $\left(\mathrm{N}_{2}\right.$ at $1100{ }^{\circ} \mathrm{C}$ for $1 \mathrm{~h}$ ) samples.

$3 \mathrm{keV}$ in the final stage. Conventional microscopy was carried out in a Philips CM 20 electron microscope operating at $200 \mathrm{kV}$, while the high resolution images were taken in a JEOL 3010 UHR microscope operating at $300 \mathrm{kV}$.

\section{RESULTS AND DISCUSSION}

The implanted samples were studied by BS in channeling geometry before and after the annealing. Figure 1(a) shows the BS/C spectra corresponding to the lowest and highest fluences implanted samples, in addition to the virgin and random spectra. As observed directly from the Si signal, the yield corresponding to the extremely damaged layer reaches the amorphous state (random level) in both cases due to the high current density $\left(2.5 \mu \mathrm{A} \mathrm{cm}^{-2}\right)$ applied during the implantation. Detailed study about the effect of the ion current density on the damage produced in the full series of implanted samples is presented in our previous work. ${ }^{3}$ The thickness of the amorphous layer is estimated as $200 \mathrm{~nm}$ for $4 \times 10^{14} \mathrm{Al} \mathrm{cm}^{-2}$ and $\approx 240 \mathrm{~nm}$ for $2 \times 10^{15} \mathrm{Al} \mathrm{cm}^{-2} \mathrm{im}$ planted $\mathrm{SiC}$, under the assumption of a material density of $3.21 \mathrm{~g} \mathrm{~cm}^{-3}$, although the real thickness is larger, since the amorphous state has a lower density.

While the crystalline structure of the lowest fluence (4 $\times 10^{14} \mathrm{Al} \mathrm{cm}^{-2}$ ) implanted sample is recovered during the subsequent annealing at $1100{ }^{\circ} \mathrm{C}$ in $\mathrm{N}_{2}$ for $1 \mathrm{~h}$, in the medium and highest fluences implanted samples still a heavily damaged layer remains, as it is observed in Fig. 1(b). However, the thickness of the damaged layer decreases due to the

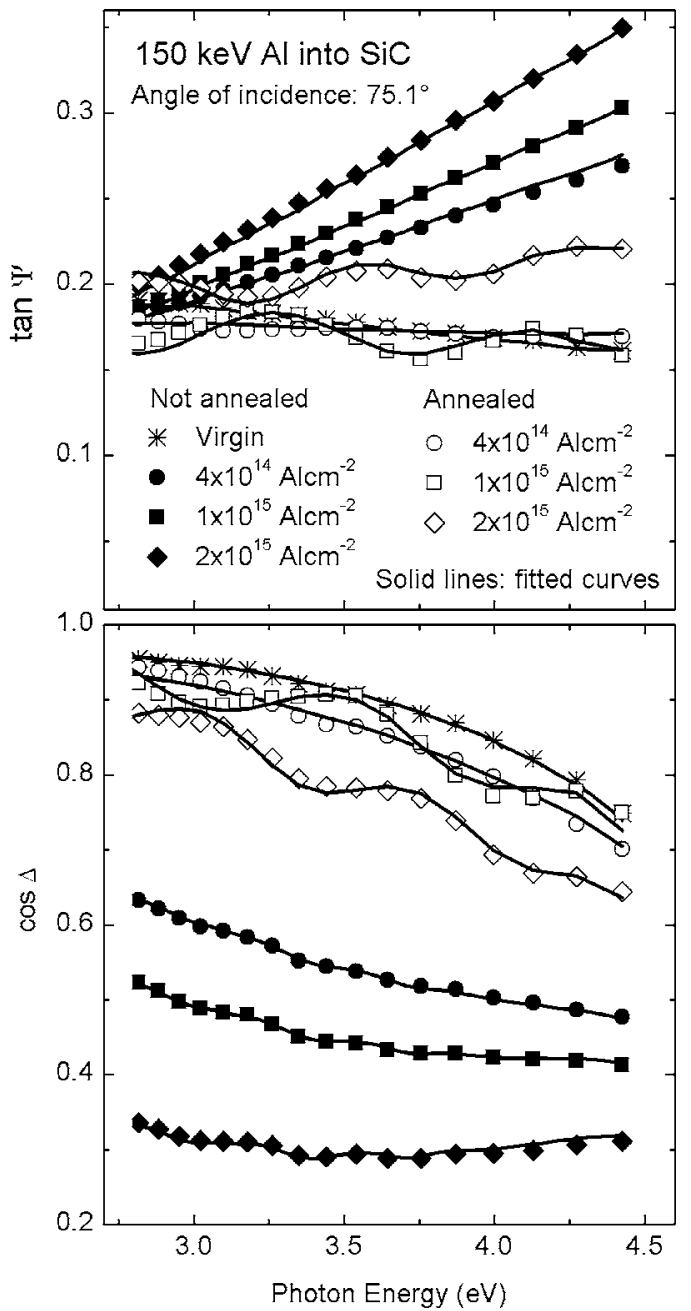

FIG. 2. Measured (symbols) and fitted (solid lines) ellipsometric data in the spectral range of $280-450 \mathrm{~nm}$. The two parameters, $\tan \Psi$ and $\cos \Delta$ are shown. The angle of incidence was $75.1^{\circ}$.

annealing process, which suggests that some recrystallization takes place. In the case of the medium fluence implanted sample $\left(1 \times 10^{15} \mathrm{Al} \mathrm{cm}^{-2}\right)$ the location of the damaged layer is just at the middle of the previous amorphous layer, from what we can consider that recrystallization started both from the substrate as well as from the surface.

On the other hand, the ellipsometric results show a systematic change for the optical parameters with the fluence before and after the annealing. The pseudo-refractive-index $(n)$ and the extinction coefficient $(k)$ increase with increasing fluence. ${ }^{5}$ In Fig. 2 a detail of the SE spectra for the asimplanted and annealed samples are plotted in the wavelength range from 280 to $450 \mathrm{~nm}$. They were well fitted by means of an optical model based on a thin surface oxide and a bulk mixture of crystalline $\mathrm{SiC}(c-\mathrm{SiC})$ and ion implantation amorphized $\mathrm{SiC}(i-a-\mathrm{SiC})$. The optical parameters for the reference $i-a-\mathrm{SiC}$ were calculated using the sample implanted with the highest dose, since this was considered to be totally amorphous. The measured spectra $(\tan \psi$ and $\cos \Delta$ versus wavelength) for the annealed samples get close to the virgin one, especially for the lower fluence. Though the corresponding to the $2 \times 10^{15} \mathrm{Al} \mathrm{cm}^{-2}$ implanted sample have interference fringes below $500 \mathrm{~nm}$ wavelength range, the op- 
TABLE I. Relative damage determined by SE, RBS, and observed by TEM.

\begin{tabular}{ccccc}
\hline \hline & & \multicolumn{3}{c}{$\begin{array}{c}\text { Relative damage } \\
(\%)\end{array}$} \\
\cline { 3 - 5 } $\begin{array}{c}\text { Fluence } \\
\left(\times 10^{15} \mathrm{Al} \mathrm{cm}^{-2}\right)\end{array}$ & Annealing & SE & RBS & TEM \\
\hline 0.4 & No & 60 & 100 & Not measured \\
0.4 & Yes & 11 & 9 & $\begin{array}{c}\text { Dislocation loops } \\
100 \% \text { amorphous } \\
1.0\end{array}$ \\
& No & 76 & 100 & strip \\
1.0 & Yes & 13 & 76 & Heavily defective \\
2.0 & No & 100 & 100 & $\begin{array}{c}\text { Not measured } \\
2.0\end{array}$ \\
\hline \hline
\end{tabular}

tical parameter values move a bit further away from the virgin. This indicates that a thin heavily damaged (or polycrystalline ${ }^{11}$ ) layer remains on the surface.

The relative damage, defined as the ratio of $i-a-\mathrm{SiC} /$ $c$-SiC, was measured using the Bruggeman effective medium approximation combining the dielectric functions of $c-\mathrm{SiC}$ (Ref. 12) and $i$ - $a$-SiC. In Table I the different relative damage values found by means of the SE and BS measurements can be compared. While SE reveals a change of the relative damage from $60 \%$ to $100 \%$ as a function of the implanted fluence, BS shows a $100 \%$ relative damage for all the asimplanted samples. After annealing SE results give the similar disorder recovery (11-13\%) for all fluences; however, BS relative damage ranges from $9 \%$ to $95 \%$. The possible explanation for this difference was presented in our earlier works. 3,5 During a postimplantation annealing of the highly damaged material, the short distance order of the material is recovered but the lost long distance order remains. It is possible that grains of different polytypes are formed and the alignments of these grains vary along the original direction. The misaligned grains are seen by BS as if they were totally amorphous, while SE sees the structure as polycrystalline.

With the help of the performed electron microscopic studies, we could confirm our predictions. Figure 3(a) shows the cross-sectional TEM image, taken in dark field mode, corresponding to the $1 \times 10^{15} \mathrm{Al} \mathrm{cm}^{-2}$ as-implanted $4 H$-SiC sample. An about $225 \mathrm{~nm}$ thick damaged region is observed, but the top region, which is about $15 \mathrm{~nm}$ thick, is still crystalline, as commonly noticed after high energy ion implantation. Both the top region of the amorphous zone and the interface region contain small crystallites left behind, which appear as bright spots in the image. Detailed HREM study revealed that the top crystalline stripe can be divided into two regions the top $10 \mathrm{~nm}$ at the surface is almost defect-free hexagonal $\mathrm{SiC}$, while the next $5 \mathrm{~nm}$ thick region is strongly defective after implantation [Fig. 3(b)]. Crystalline SiC grains can be easily observed and found in high density to the depth of $40 \mathrm{~nm}$ [Fig. 3(b)]. The middle of the damaged zone is completely amorphous. The bottom interface of the amorphous/crystalline $\mathrm{SiC}$ shows a sharp interface on the HREM images [Fig. 3(c)] with steps in the crystalline region with about $2 \mathrm{~nm}$ height typically.

Figure 4 shows the microstructure of three samples implanted with different fluences and annealed at $1100{ }^{\circ} \mathrm{C}$ for $1 \mathrm{~h}$ in $\mathrm{N}_{2}$. Already the results of BS/C and SE investigations
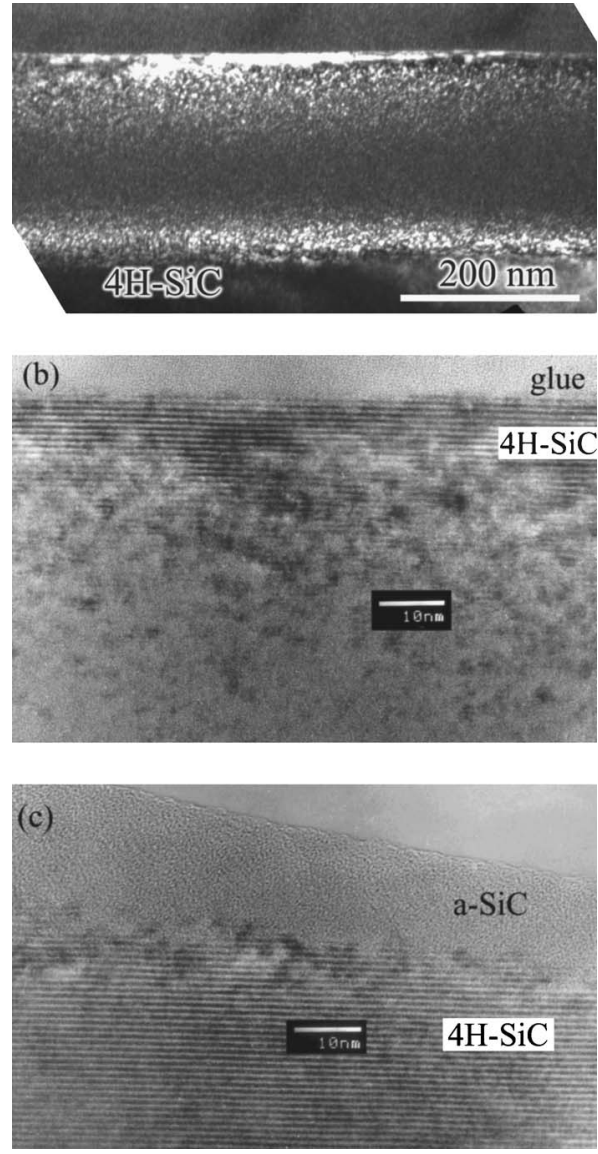

FIG. 3. Cross-sectional images taken on the $1 \times 10^{15} \mathrm{Al} \mathrm{cm}^{-2}$ as-implanted $4 H$-SiC sample. (a) Dark field image showing the whole implanted region, (b) high resolution image taken on the surface region, and (c) high resolution image taken at the end of implantation range showing the amorphized/ crystalline interface.

show that the structural recovery is proportional to the implanted fluence. In the case of the lowest fluence implanted sample the structure is crystalline after annealing [Fig. 4(a)]. Traces of ion implantation are still visible to the depth of about $180 \mathrm{~nm}$ after annealing. However, the top $40 \mathrm{~nm}$ thick region is high quality single crystal without extended defects (TEM is not sensitive for point defects). The contrast of the defective stripe [Fig. 4(a)] is very characteristic for small dislocation loops of extrinsic type. It is already known ${ }^{13}$ that $\mathrm{Al}$ is not concentrated in the loops, therefore those were formed by $\mathrm{Si}$ accumulation. This is attributed to activation of the $\mathrm{Al}$ atoms by taking substitutional sites creating $\mathrm{Si}$ interstitial. Annealing of the medium dose implanted sample resulted in the structure shown in Fig. 4(b). The implanted zone is already crystalline but a heavily damaged zone is formed during recrystallization, that extends to the depth of about $200 \mathrm{~nm}$. There are less defects in the top $45 \mathrm{~nm}$ region, but that is far more defective than the appropriate region of our low fluence implanted sample.

The sample implanted to the highest fluence is also crystalline after annealing [Fig. 4(c)] with a $225 \mathrm{~nm}$ thick top region, which is defective. However, there are small and large inclusions which are cubic $\mathrm{SiC}$ grains. One of the large grains in the upper half of the recrystallized zone is labeled as $3 C$. Bright field and dark field images were taken in the 

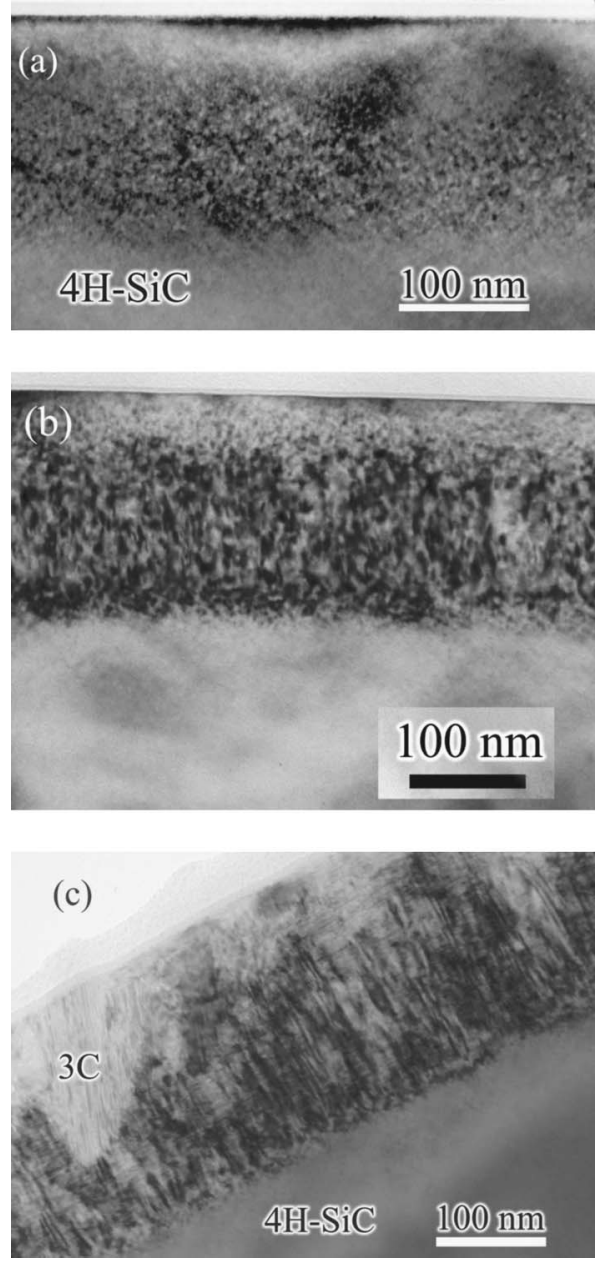

FIG. 4. Cross-sectional TEM images corresponding to the annealed $4 H$ SiC samples: (a) $4 \times 10^{14} \mathrm{Al} \mathrm{cm}^{-2}$, (b) $1 \times 10^{15} \mathrm{Al} \mathrm{cm}^{-2}$, and (c) 2 $\times 10^{15} \mathrm{Al} \mathrm{cm}^{-2}$.

TEM, which made possible the selection of cubic $\mathrm{SiC}$ grains. The amount of $3 C-\mathrm{SiC}$ grains is estimated to about $30 \%$ in the near surface region. The high resolution image in Fig. 5 shows a part of the sample just below the surface, with one large $3 C$-SiC grain. The grain forms a $\mathrm{V}$ shape in cross section (the boundary is marked by a dashed line), in which two different $3 C$-SiC grains are formed in a twinned configuration. Both of the cubic and the hexagonal $\mathrm{SiC}$ regions in the image are very defective. The TEM results are also included in Table I for comparison to the results of the SE and RBS measurements.

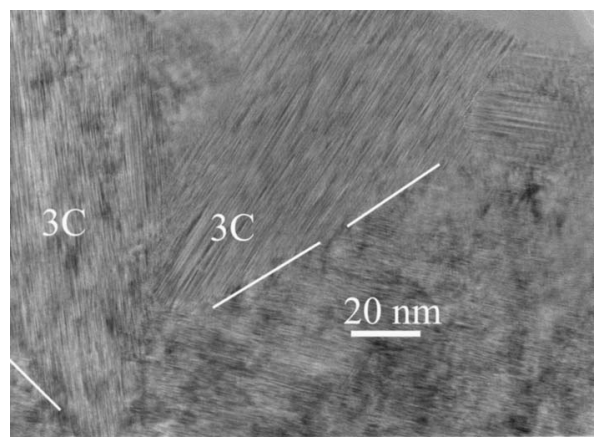

FIG. 5. HREM image from a region of the $2 \times 10^{15} \mathrm{Al} \mathrm{cm}^{-2}$ implanted $4 H$-SiC sample after annealing. Different $\mathrm{SiC}$ polytypes can be observed.
All of our as-implanted $\mathrm{SiC}$ samples contained a heavily damaged zone. However, the top region of the samples implanted to the lowest and to the medium fluence was crystalline. During annealing the solid phase regrowth of $\mathrm{SiC}$ started from two directions, i.e., from the crystalline surface and interface regions. The structural quality of the originally crystalline near surface region was even further improved during the annealing process because some of the defects were annealed out. In our highest fluence $\left(2 \times 10^{15}\right.$ $\mathrm{Al} \mathrm{cm}-2$ ) implanted sample the damage extended to the top surface. Therefore the crystallization during annealing started as the growth of hexagonal region from the amorphous/crystalline interface and started by the nucleation of crystalline grains in the top amorphous region. The temperature of annealing is relatively low, at which the cubic polytype is nucleated preferentially and this gives an explanation for the appearance of cubic grains in the sample.

\section{CONCLUSIONS}

The study carried out by transmission microscopy can explain the different results found by backscattering and ellipsometry, corroborating our previous hypothesis. In a highly damaged $\mathrm{SiC}$ crystal, the short distance order is achieved during the postimplantation annealing, but not the long distance order. The recovery of the short distance order in the implanted material during the annealing process takes place in a parallel way from the surface and the bulk of the substrate. If the lattice disorder is small enough, the original crystalline structure may be recovered in the doped region. When the amorphized region extends to the top surface cubic inclusions are formed during annealing at $1100{ }^{\circ} \mathrm{C}$.

\section{ACKNOWLEDGMENTS}

This work is partially supported by the Spanish MCyT (Project No. MAT2002-02843, 70\% FEDER funds), by the Spanish-Hungarian Scientific Collaboration Agreement No. E27/2001, and by the Hungarian OTKA Grant Nos. T032029, T047011, T043704, and K61725. One of the authors (J.G.L.) acknowledges the "Ramón y Cajal" program of the Spanish MCyT for financial support. Two of the authors (P.P.) and (B.P.) are grantee of the Bolyai János Scholarship.

${ }^{1}$ J. W. Palmour, J. A. Edmond, H. S. Kong, and C. H. Carter, Jr., Physica B 185, 461 (1993).

${ }^{2}$ G. Battistig, J. García López, N. Q. Khánh, Y. Morilla, M. A. Respaldiza, and E. Szilágyi, Mater. Sci. Forum 433-436, 625 (2003).

${ }^{3}$ G. Battistig, J. García López, Y. Morilla, N. Q. Khánh, T. Lohner, P. Petrik, and A. R. Ramos, Nucl. Instrum. Methods Phys. Res. B 219-220, 652 (2004).

${ }^{4}$ A. Hallén et al., Nucl. Instrum. Methods Phys. Res. B 186, 186 (2002).

${ }^{5}$ P. Petrik et al., Thin Solid Films 455-456, 239 (2004).

${ }^{6}$ E. R. Shaaban, T. Lohner, P. Petrik, N. Q. Khánh, M. Fried, O. Polgár, and J. Gyulai, Phys. Status Solidi A 195, 277 (2003).

${ }^{7}$ N. Q. Khánh, Z. Zolnai, T. Lohner, L. Tóth, L. Dobos, and J. Gyulai, Nucl. Instrum. Methods Phys. Res. B 161-163, 424 (2000).

${ }^{8}$ W. Fukarek, R. A. Yankov, W. Anwand, and V. Heera, Nucl. Instrum. Methods Phys. Res. B 142, 570 (1998). 
${ }^{9}$ Z. Zolnai, N. Q. Khánh, E. Szilágyi, E. Kótai, A. Ster, M. Posselt, T. Lohner, and J. Gyulai, Diamond Relat. Mater. 11, 1239 (2002).

${ }^{10}$ E. Kótai, Nucl. Instrum. Methods Phys. Res. B 85, 588 (1994).

${ }^{11}$ R. Nipoti and A. Parisini, Philos. Mag. B 80, 647 (2000).
${ }^{12}$ S. Zollner, J. G. Chen, E. Duda, T. Wetteroth, S. R. Wilson, and J. N. Hilfiker, J. Appl. Phys. 85, 8353 (1999).

${ }^{13}$ P. O. A. Persson, L. Hultman, M. S. Janson, A. Hallen, R. Yakimova, D. Panknin, and W. Skorupa, J. Appl. Phys. 92, 2501 (2002). 